\title{
Use of the Environment and Policy Evaluation and Observation as a Self-Report Instrument (EPAO-SR) to measure nutrition and physical activity environments in child care settings: validity and reliability evidence
}

\author{
Dianne S. Ward ${ }^{1 *}$, Stephanie Mazzucca ${ }^{2}$, Christina McWilliams ${ }^{3}$ and Derek Hales ${ }^{2}$
}

\begin{abstract}
Background: Early care and education (ECE) centers are important settings influencing young children's diet and physical activity (PA) behaviors. To better understand their impact on diet and PA behaviors as well as to evaluate public health programs aimed at ECE settings, we developed and tested the Environment and Policy Assessment and Observation - Self-Report (EPAO-SR), a self-administered version of the previously validated, researcher-administered EPAO.

Methods: Development of the EPAO-SR instrument included modification of items from the EPAO, community advisory group and expert review, and cognitive interviews with center directors and classroom teachers. Reliability and validity data were collected across 4 days in 3-5 year old classrooms in 50 ECE centers in North Carolina. Center teachers and directors completed relevant portions of the EPAO-SR on multiple days according to a standardized protocol, and trained data collectors completed the EPAO for 4 days in the centers. Reliability and validity statistics calculated included percent agreement, kappa, correlation coefficients, coefficients of variation, deviations, mean differences, and intraclass correlation coefficients (ICC), depending on the response option of the item.

Results: Data demonstrated a range of reliability and validity evidence for the EPAO-SR instrument. Reporting from directors and classroom teachers was consistent and similar to the observational data. Items that produced strongest reliability and validity estimates included beverages served, outside time, and physical activity equipment, while items such as whole grains served and amount of teacher-led PA had lower reliability (observation and self-report) and validity estimates. To overcome lower reliability and validity estimates, some items need administration on multiple days.

Conclusions: This study demonstrated appropriate reliability and validity evidence for use of the EPAO-SR in the field. The self-administered EPAO-SR is an advancement of the measurement of ECE settings and can be used by researchers and practitioners to assess the nutrition and physical activity environments of ECE settings.
\end{abstract}

Keywords: Child care, Preschoolers, Nutrition, Physical activity, Measurement

\footnotetext{
* Correspondence: dsward@email.unc.edu

${ }^{1}$ Department of Nutrition, Gillings School of Global Public Health, University of North Carolina at Chapel Hill, 2207 McGavran-Greenberg Hall, CB 7461,

Chapel Hill, NC 27599-7461, USA

Full list of author information is available at the end of the article
} 


\section{Introduction}

Childhood obesity remains a serious public health concern, with about $25 \%$ of children 2-5 years old in the United States (US) classified as overweight or obese (BMI $\geq 85$ th percentile) [1]. Excess weight in childhood increases the risk of a child becoming overweight or obese in adulthood [2] and suffering from other chronic health conditions [3-6]. Early childhood has been identified as a critical period for the development of obesity [7-9] and thus, an important target for nutrition and physical activity programs [10].

Child care centers are a particularly important setting to help shape life-long diet and physical activity (PA) behaviors for the prevention of childhood overweight [11]. Recent estimates show that $61 \%$ of preschool aged children attend a center-based child care program, where they spend, on average, $25-30 \mathrm{~h}$ per week [12]. According to the American Dietetic Association, children receive $1 / 2-2 / 3$ of their daily recommended calories while in full time child care [13]. Also, national standards in Caring for Our Children recommend that young children receive 90-120 min of moderate- to- vigorous physical activity (MVPA) per 8-hour day in child-care [14]. However, research shows that often children in child care are not meeting nutrition recommendations $[15,16]$ and that PA levels are low [17]. Experts have called for the development of child care-based interventions to promote better nutrition and PA behaviors and have stressed the importance of comprehensive interventions that target nutrition- and physical activity-related policies and practices [11].

In order to evaluate such interventions, an accurate, comprehensive assessment of the nutrition and PA environments at child care settings is vital. Currently, appropriate measures to assess these environments are limited [18]. One tool that does exist is the Environment and Policy Assessment and Observation (EPAO) instrument [19]. The EPAO was originally created to evaluate the Nutrition and Physical Activity Self-Assessment for Child Care (NAP SACC) intervention, which is based on a set of best practice recommendations. These best practices were developed through an extensive review of all existing national (United States) recommendations and research evidence for healthy nutrition and physical activity environments in center-based child care, and was reviewed by experts in health and child care [20-23]. Although developed for a specific initiative, the EPAO has been utilized widely by researchers because of its comprehensive scope and its link to evidence-based practice [24-32]. The EPAO is an intensive, day-long environmental observation that evaluates all of the provisions and practices occurring within centers and includes a document review of center-based policies. Outcomes from the EPAO provide a comprehensive measure of nutrition and PA environmental characteristics of a child care center, and the instrument has been shown to have strong interobserver agreement [19].

In addition to the EPAO, two other instruments exist to assess the nutrition and physical activity characteristics at child care centers, but these either evaluate only written policies (WellCCAT), or are limited to physical activity/ weather policies (ELEPhANTS). The Wellness Child Care Assessment Tool (WellCCAT) is a 65-item survey completed by center directors about policies (e.g., nutrition education, food/beverage standards, and physical activity) contained in a center's written documents [33]. The Early Learning Environments for Physical Activity and $\mathrm{Nu}-$ trition Environments Telephone Survey (ELEPhANTS) is a telephone-administered center director interview to assess aspects of a center's physical activity practices and includes items about weather and clothing policies, playground size, access, surfaces, shade, topography, and quality; program structure; staff training and behavior; and the sedentary environment [34].

Because the observation format of the EPAO can be costly to implement and requires considerable effort to train and certify data collectors, we modified the EPAO instrument to be completed by center staff using a selfreport format (EPAO-SR). To our knowledge, only one selfadministered instrument is available to assess both nutrition and physical activity environments [35]. In that survey, directors are asked to report on their center's nutrition and physical activity practices (23 items), physical environment (3 items, including 11 types of equipment, books and posters), and policies (9 items), as well as the nutrition quality of foods and beverages served ( 36 items). Evidence used to substantiate validity for most of the items in this instrument was either an interview with the same director who completed the self-report or printed menus; observation by research staff was used to validate only 9 of the survey's items. We felt that there was a need for a more comprehensive instrument to characterize the nutrition and physical activity environments at child care centers based on strong reliability and validity evidence. Thus, we present the EPAO-SR in this paper, including the instrument's development, methods for establishing psychometric properties, reliability and validity data, and recommendations for using the new EPAO-SR.

\section{Methods}

\section{EPAO-SR instrument development}

Development of the EPAO-SR instrument occurred in three steps: 1) modification of items from the existing observation-based instrument, 2) review by community advisory group and experts, and 3) cognitive interviews with center directors and classroom teachers.

Development began with a thorough review of the original EPAO by our research group. Items were modified from an observation to a self-report format and questions 
were added based on the current literature. Additionally, modifications were made to improve item clarity and comprehension based on our extensive field experience with the EPAO and the inter-observer agreement data obtained when the tool was first developed [19]. The SR-based instrument was then distributed to our child care community advisory committee and to two national experts for review. Our advisory committee is comprised of child care professionals, local and state health officials, extension agents, child care providers, and parents, and meets annually to guide NAP SACC and other early care and education activities. The advisory committee and experts were asked to comment on content coverage, and item relevance, format and clarity. Revisions were made based on the feedback from the reviewers.

Finally, cognitive interviews were conducted with a convenience sample of 35 center directors and classroom teachers from across North Carolina. A cognitive interview is a technique commonly used in survey questionnaire development to ensure that the questionnaire designer's intended interpretation comes across clearly to the respondent and increases the probability that they will respond in a thoughtful manner and give accurate answers [36]. Directors and teachers had participated in a previous study with our research group. Directors and teachers received a subset of the questions by mail prior to the interview and were paid $\$ 25$ for participating. During the 1-hour telephone interview, trained research staff conducted guided cognitive interviews using a set of questions and prompts developed by our team. Probes from the initial sets of interviews focused on understanding of item content, mental processes used to respond to certain items, and obvious omissions. Later interviews focused more on the layout, structure, and organization of the self-report instrument. Content analysis of the interviews occurred in an iterative manner. When five interviews were completed for a section of questions, issues were summarized and the project team discussed the problems. Revisions were made as needed and questions were reassessed. After three rounds of interviews and revisions the instrument was considered acceptable for further testing.

\section{Instrument}

The EPAO-SR was designed to evaluate a center's provisions (foods/beverages served, active \& sedentary opportunities, PA equipment and the outdoor environment), practices (nutrition and physical activity social environment), and policies (both nutrition and PA). The EPAOSR contains close to 800 items. An overview of the items is found in Table 1. Based on experience in the development of the original EPAO instrument and feedback from our advisory committee, we felt that it would be necessary to obtain data from both directors and teachers to obtain the most accurate description of the child care centers' nutrition and physical activity environments. The selfreporting version (EPAO-SR) is divided into three surveys: the Director Report (completed by the director), the Staff Daily Questionnaire and the Staff General Questionnaire

Table 1 Overview of the EPAO and EPAO-SR and Field Testing Summary

\begin{tabular}{|c|c|c|c|c|c|c|c|}
\hline \multirow[t]{2}{*}{ Category } & \multirow[t]{2}{*}{ Sample description of items } & \multirow[t]{2}{*}{ Questionnaire } & \multirow[t]{2}{*}{ Reporter } & \multicolumn{4}{|c|}{ Day completed } \\
\hline & & & & 1 & 2 & 3 & 4 \\
\hline \multirow{3}{*}{$\begin{array}{l}\text { Provisions 1: food and } \\
\text { beverages served; active play } \\
\text { and sedentary opportunities }\end{array}$} & - Types of food and beverages served & Staff today survey & Classroom teachers & $x$ & $x$ & $x$ & $X$ \\
\hline & $\begin{array}{l}\text { - Amount of active play time offered, indoor } \\
\text { and outdoor }\end{array}$ & Daily observation tool & Research staff & $x$ & $x$ & $x$ & X \\
\hline & - Amount of TV, computer, and seated time & & & & & & \\
\hline \multirow[t]{3}{*}{$\begin{array}{l}\text { Provisions 2: classroom } \\
\text { physical environment }\end{array}$} & $\begin{array}{l}\text { - Classroom posters or books featuring } \\
\text { food or PA }\end{array}$ & Staff general survey & Classroom teachers & $x$ & & $X$ & \\
\hline & - Fixed and portable play equipment & Daily observation tool & Research staff & $x$ & $x$ & $X$ & X \\
\hline & - Amount of space for active play & & & & & & \\
\hline \multirow[t]{2}{*}{$\begin{array}{l}\text { Provisions 3: center's } \\
\text { physical environment }\end{array}$} & $\begin{array}{l}\text { - Natural features, e.g., trees, open play } \\
\text { space }\end{array}$ & Director survey & Center director & $x$ & & $x$ & \\
\hline & - Presence of vending machines & Daily observation tool & Research staff & $x$ & $x$ & $x$ & $x$ \\
\hline \multirow{2}{*}{$\begin{array}{l}\text { Practices: teacher } \\
\text { engagement with children } \\
\text { around eating \& activity }\end{array}$} & $\begin{array}{l}\text { - Teacher behavior around mealtimes and } \\
\text { active play time periods }\end{array}$ & Staff today survey & Classroom teachers & $x$ & & $x$ & \\
\hline & $\begin{array}{l}\text { - Use of food or PA as a reward or } \\
\text { punishment }\end{array}$ & Daily observation tool & Research staff & X & $x$ & $x$ & $x$ \\
\hline \multirow{3}{*}{$\begin{array}{l}\text { Policies, Training and } \\
\text { Education: regulations, } \\
\text { planned trainings, \& formal } \\
\text { education }\end{array}$} & - Policies regarding nutrition or PA & Director survey & Center director & $x$ & & $x$ & \\
\hline & $\begin{array}{l}\text { - Nutrition- or PA-related training for center } \\
\text { staff }\end{array}$ & Document review & Research staff & & & & $X$ \\
\hline & $\begin{array}{l}\text { - Parent education around nutrition or PA, } \\
\text { e.g., workshops, emails, pamphlets }\end{array}$ & & & & & & \\
\hline
\end{tabular}


(both staff questionnaires completed by teachers). Each survey contained questions that would be most appropriate for the individual (either director or teacher) to answer. The Director Report asks directors about center-wide nutrition and PA efforts, including parent education and policies for nutrition and PA. The Staff Daily Questionnaire asks classroom teachers to report on daily nutrition and PA provisions and practices on a specific day in a manner similar to a time use diary, while the Staff General Questionnaire asks teachers to report generally on their nutrition and PA practices and infrequent activities such as participation in nutrition and PA training. Most items were presented in a checklist format such that items, or sections, could be skipped if certain activities did not occur or certain types of foods were not served.

\section{Reliability and validity testing \\ Sample}

Child care centers with at least a 2-star rating (North Carolina quality improvement $1-5$ star rating scale) were recruited across six counties in piedmont North Carolina via mailed flyers and phone calls from research staff. Eligible centers were identified using the North Carolina Division of Child Development and Early Education website (http://ncchildcare.dhhs.state.nc.us/general/home.asp). Center demographics were obtained during the screening call with the center director. Consent forms were mailed to centers and collected by mail or during the first observation visit (see below.) Directors gave consent for center participation in the study, and parents gave consent for their children to wear accelerometers during the observation period (data not included in this paper). Centers received \$100 as an incentive for participation and for completing measures. All methods were reviewed and approved by the University of North Carolina Institutional Review Board.

\section{Data collection procedures}

A summary of field testing procedures can be found in Table 2. Data were collected across 4 days in each center. Directors identified teachers of classrooms with 3-5 year olds to complete questionnaires and allowed research staff to observe these classrooms for four consecutive days. At least one teacher per center completed the survey, and additional teachers completed the survey if there were multiple teachers per classroom or multiple 3-5 year old classrooms in a center. During the 4-day data collection, classroom teachers completed the Staff Daily Questionnaire for each of the 4 days and the Staff General Questionnaire on two non-consecutive days. Also, directors completed the Director Report on two non-consecutive days. Directors and teachers completed a demographic questionnaire on the first observation day.

Research staff trained and certified to conduct the EPAO (observation format) completed direct observations of
Table 2 Participant characteristics

\begin{tabular}{|c|c|c|}
\hline Characteristic & $\begin{array}{l}\text { Centers } \\
(n=50) \\
\end{array}$ & \\
\hline Median star rating ${ }^{a}$ & 4 & \\
\hline Mean years in operation & 16 & \\
\hline CACFP ${ }^{\mathrm{b}}$ participation (\%) & 46 & \\
\hline Mean enrollment (\#) & 78 & \\
\hline Children receive subsidies (\%) & 52 & \\
\hline \multicolumn{3}{|l|}{ Race/ethnicity of children (\%) } \\
\hline Non-Hispanic White & 57 & \\
\hline Non-Hispanic Black & 31 & \\
\hline \multirow[t]{2}{*}{ Hispanic } & 8 & \\
\hline & $\begin{array}{l}\text { Directors } \\
(n=50)\end{array}$ & $\begin{array}{l}\text { Teachers } \\
(n=124)\end{array}$ \\
\hline Time as center director (years) & 10 & \\
\hline Time as classroom teacher (years) & & 10 \\
\hline Age (years) & 44 & 37 \\
\hline Females (\%) & 96 & 100 \\
\hline \multicolumn{3}{|l|}{ Highest level of education (\%) } \\
\hline High school or lower & 0 & 10 \\
\hline Some college & 25 & 39 \\
\hline Associates degree & 23 & 22 \\
\hline Bachelor's degree & 40 & 25 \\
\hline Masters/Doctoral degree & 13 & 3 \\
\hline \multicolumn{3}{|l|}{ BMI Category (\%) } \\
\hline Underweight or normal $(<25)$ & 35 & 42 \\
\hline Overweight (25-29.9) & 23 & 22 \\
\hline Obese $(\geq 30)$ & 42 & 36 \\
\hline \multicolumn{3}{|l|}{ Race/ethnicity (\%) } \\
\hline Non-Hispanic White & 60 & 53 \\
\hline Non-Hispanic Black & 36 & 39 \\
\hline Hispanic & 2 & 2 \\
\hline
\end{tabular}

${ }^{\mathrm{a}} \mathrm{NC}$ Quality Rating System (1-5 stars)

${ }^{\mathrm{b}}$ CACFP Child and adult care food program

classrooms with 3-5 year olds for four consecutive days. Observations occurred from the start of the first meal until the majority of the children had left each day. Most observations $(n=48)$ were conducted using one data collector per center. For the remaining two centers, two data collectors conducted the 4-day observations, each observing for 2 days. Documents including policy handbooks, training certificates, menus, and parent education materials were collected from the director for the Document Review. Three data collectors were trained during an intensive halfday workshop that included a review of EPAO items and study protocols, and individuals were certified through an additional half-day observation in a practice center. Percent 
agreement was calculated between each data collector and the gold standard observer (member of the research team). Staff were certified if agreement was at least $85 \%$ with the gold standard.

\section{Statistical analysis}

Because of the variation in item response format (i.e., continuous or categorical) for the EPAO and EPAO-SR, statistics used to assess reliability and validity evidence at the item level varied. ANOVAs were used to test differences in means across days (e.g., mean of fruit served for days 1,2 , 3 , and 4 of observation data), with p-values greater than 0.05 indicating no significant difference of means across days. P-values for these tests are presented in Tables 3, 4, 5, 6 and 7. The following were used to evaluate the evidence where appropriate: percent agreement, kappa, correlation coefficients, coefficients of variation, deviations, mean differences, and intraclass correlation coefficients (ICC). Scale level data were evaluated based on ICCs. Stability of item and scale scores from the new self-report instrument over multiple days was determined by computing reliability statistics for item and scale scores from the self-report across multiple days. Two ICCs are reported, one represents the reliability if all days of available data are averaged to compute an item score (ICC4 or ICC2), the other (ICC1) tells us the reliability if one randomly selected day was used to represent a center's score for an item. For nutrition and physical activity policies, two-level (no written policy vs. written policy present) percent agreement and Kappa statistics were calculated to compare research staff and center director reports of policies. We used Shrout's categorizations to evaluate ICC and Kappa statistics: virtually none $(0-0.10)$, slight $(0.11-0.40)$, fair $(0.41-$ $0.60)$, moderate $(0.61-0.8)$, and substantial $(0.81-1.0)$ [37]. Analyses were conducted using SAS v9.2 (Cary, NC).

\section{Results}

\section{Sample characteristics}

2A sample of 50 child care centers, 50 center directors, and 124 classroom teachers participated in the study. Characteristics of participating centers, directors, and teachers are described in Table 2. Briefly, $46 \%$ of centers participated in the Child and Adult Care Food Program (CACFP) and $52 \%$ enrolled children who received subsidies towards their enrollment fees. About half (57\%) of children attending the centers were Non-Hispanic White, $31 \%$ were Non-Hispanic Black and $8 \%$ were Hispanic. Center directors were on average 44 years old, and classroom teachers' average age was 37 years. About half of directors (53\%) had received a bachelor's degree or higher, while only

Table 3 Food and beverages served - means, reliability, and validity

\begin{tabular}{|c|c|c|c|c|c|c|c|c|c|}
\hline \multirow[b]{2}{*}{ Food or beverage provision } & \multirow[b]{2}{*}{ Source } & \multirow[b]{2}{*}{$\begin{array}{l}\text { Means (range day } \\
1-4)\end{array}$} & \multirow[b]{2}{*}{$\begin{array}{l}p \text {-value } \\
\text { means }\end{array}$} & \multicolumn{2}{|c|}{ Reliability } & \multicolumn{4}{|l|}{ Validity } \\
\hline & & & & $\begin{array}{l}\text { ICC } \\
\text { 1-day }\end{array}$ & $\begin{array}{l}\text { ICC } \\
\text { 4-day }\end{array}$ & $\begin{array}{l}\text { Corr } \\
\text { (days) }\end{array}$ & $\begin{array}{l}\text { Corr } \\
\text { (avg) }\end{array}$ & Avg dev & $\begin{array}{l}\text { Dev as } \\
\% \text { of mean }\end{array}$ \\
\hline \multicolumn{10}{|l|}{ Food } \\
\hline \multirow[t]{2}{*}{ Total grains } & Obs & $2.75-2.96$ & 0.49 & 0.16 & 0.42 & $0.14-0.35$ & 0.25 & 0.20 & $7.9 \%$ \\
\hline & Avg staff & $2.27-2.73$ & 0.02 & 0.25 & 0.57 & & & & \\
\hline \multirow[t]{2}{*}{ Meat or alternative } & Obs & $1.00-1.33$ & 0.15 & 0.14 & 0.40 & $0.05-0.61$ & 0.32 & 0.01 & $1.2 \%$ \\
\hline & Avg staff & $1.01-1.09$ & 0.95 & 0.24 & 0.56 & & & & \\
\hline \multirow[t]{2}{*}{ Fruit (not juice) } & Obs & $1.38-1.71$ & 0.11 & 0.28 & 0.61 & $0.05-0.64$ & 0.53 & 0.13 & $9.2 \%$ \\
\hline & Avg staff & $1.20-1.48$ & 0.38 & 0.32 & 0.65 & & & & \\
\hline \multirow[t]{2}{*}{ Vegetables (non-potatoes) } & Obs & $0.76-0.98$ & 0.13 & 0.07 & 0.22 & $0.29-0.63$ & 0.40 & 0.02 & $2.7 \%$ \\
\hline & Avg staff & $0.71-0.94$ & 0.15 & 0.06 & 0.20 & & & & \\
\hline \multicolumn{10}{|l|}{ Beverages } \\
\hline \multirow[t]{2}{*}{ Milk } & Obs & $1.91-2.10$ & 0.62 & 0.32 & 0.65 & $0.60-0.69$ & 0.85 & 0.05 & $2.4 \%$ \\
\hline & Avg staff & $1.90-1.93$ & 0.97 & 0.45 & 0.77 & & & & \\
\hline \multirow[t]{2}{*}{100 \% Fruit Juice (oz) } & Obs & $3.02-3.80$ & 0.77 & 0.38 & 0.71 & $0.36-0.53$ & 0.51 & -0.77 & $18.0 \%$ \\
\hline & Avg staff & $3.55-4.61$ & 0.37 & 0.36 & 0.70 & & & & \\
\hline \multirow[t]{2}{*}{ Water (oz) } & Obs & $3.12-3.35$ & 1.00 & 0.61 & 0.86 & $0.48-0.58$ & 0.63 & -0.60 & $14.9 \%$ \\
\hline & Avg staff & $3.29-4.00$ & 0.78 & 0.55 & 0.83 & & & & \\
\hline
\end{tabular}

All provisions are number of offerings, except FJ and water in ounces

$P$-values represent a statistical comparison of the equivalence of means for each day of reporting (means on days $1-4)$ for each reporter. $P$-values $>0.05$ indicate no statistical difference between means across days

ICC Intraclass correlation, Corr Pearson correlation,

Dev Difference (observation - staff report) 
Table 4 Physical activity and sedentary opportunities - means, reliability, and validity

\begin{tabular}{|c|c|c|c|c|c|c|c|c|c|}
\hline \multirow[b]{2}{*}{ Physical activity provision } & \multirow[b]{2}{*}{ Source } & \multirow[b]{2}{*}{$\begin{array}{l}\text { Means (range } \\
\text { day 1-4) }\end{array}$} & \multirow[b]{2}{*}{$\begin{array}{l}p \text {-value } \\
\text { means }\end{array}$} & \multicolumn{2}{|c|}{ Reliability } & \multicolumn{4}{|c|}{$\underline{\text { Validity }}$} \\
\hline & & & & ICC1 & ICC4 & $\begin{array}{l}\text { Corr } \\
\text { (avg) }\end{array}$ & $\begin{array}{l}\text { Corr } \\
\text { (days) }\end{array}$ & $\begin{array}{l}\text { Avg } \\
\text { dev }\end{array}$ & $\begin{array}{l}\text { Dev as \% of } \\
\text { mean }\end{array}$ \\
\hline \multicolumn{10}{|l|}{ Outside } \\
\hline \multirow[t]{2}{*}{ Time outside (min) } & Obs & $69.4-74.5$ & 0.46 & 0.23 & 0.54 & 0.63 & $0.44-0.77$ & 5.8 & 9.0 \\
\hline & Avg staff & $58.7-70.4$ & 0.33 & 0.55 & 0.83 & & & & \\
\hline \multirow[t]{2}{*}{ Teacher-lead activity (bouts) } & Obs & $0.8-1.1$ & 0.64 & 0.13 & 0.37 & 0.10 & $-0.03-0.29$ & -1.2 & 54.5 \\
\hline & Avg staff & $2.1-2.7$ & 0.44 & 0.24 & 0.56 & & & & \\
\hline \multicolumn{10}{|l|}{ Inside } \\
\hline \multirow[t]{2}{*}{ Teacher-led activity (bouts) } & Obs & $1.1-1.4$ & 0.72 & 0.25 & 0.58 & 0.05 & $0.02-0.09$ & -1.6 & 57.1 \\
\hline & Avg staff & $2.4-3.0$ & 0.91 & 0.24 & 0.55 & & & & \\
\hline \multirow[t]{2}{*}{ Teacher-led activity (min) } & Obs & $7.5-11.7$ & 0.44 & 0.11 & 0.32 & 0.07 & $0.00-0.13$ & -22.3 & 71.7 \\
\hline & Avg staff & $23.1-42.0$ & 0.58 & 0.19 & 0.48 & & & & \\
\hline \multirow[t]{2}{*}{ Music and dance (min) } & Obs & $4.8-7.4$ & 0.60 & 0.22 & 0.53 & 0.32 & $0.01-0.40$ & -5.7 & 47.9 \\
\hline & Avg staff & $9.5-14.3$ & 0.07 & 0.34 & 0.67 & & & & \\
\hline \multirow[t]{2}{*}{ Gross motor activity (min) } & Obs & $2.2-5.3$ & 0.45 & 0.28 & 0.61 & 0.23 & $0.15-0.44$ & -3.3 & 40.7 \\
\hline & Avg staff & $6.4-9.9$ & 0.29 & 0.28 & 0.61 & & & & \\
\hline \multirow[t]{2}{*}{ TV time (min) } & Obs & $5.1-8.7$ & 0.84 & 0.39 & 0.72 & 0.47 & $0.24-0.71$ & 3.1 & 84.0 \\
\hline & Avg staff & $2.3-4.5$ & 0.61 & 0.41 & 0.73 & & & & \\
\hline \multirow[t]{2}{*}{ Seated time (min) } & Obs & $47.9-54.3$ & 0.83 & 0.53 & 0.82 & -0.01 & $-0.10-0.12$ & 13.1 & 40.3 \\
\hline & Avg staff & $25.4-38.2$ & 0.01 & 0.37 & 0.70 & & & & \\
\hline
\end{tabular}

$P$-values represent a statistical comparison of the equivalence of means for each day of reporting (means on days $1-4)$ for each reporter. $P$-values $>0.05$ indicate no statistical difference between means across days.

ICC Intraclass correlation,

Corr Pearson correlation

Dev Difference (observation - staff report)

Table 5 Physical activity equipment and natural environment - means, reliability, and validity

\begin{tabular}{|c|c|c|c|c|c|c|c|c|c|c|}
\hline \multirow[b]{2}{*}{ PA environment } & \multirow[b]{2}{*}{ Source } & \multicolumn{3}{|c|}{ Means } & \multicolumn{2}{|c|}{ Reliability } & \multicolumn{4}{|c|}{ Validity } \\
\hline & & D1 & D2 & $p$-value means & ICC1 & ICC2 & $\begin{array}{l}\text { Corr } \\
\text { (avg) }\end{array}$ & Corr (days) & Avg dev & $\begin{array}{l}\text { Dev as \% } \\
\text { of mean }\end{array}$ \\
\hline \multirow[t]{2}{*}{ Natural elements } & Obs & 2.7 & 2.8 & 0.99 & 0.93 & 0.96 & 0.70 & $0.65-0.77$ & -1.50 & 35.3 \\
\hline & Dir & 4.2 & 4.3 & 0.83 & 0.94 & 0.97 & & & & \\
\hline \multirow[t]{2}{*}{ Fixed equipment } & Obs & 6.9 & 7.0 & 0.73 & 0.98 & 0.99 & 0.51 & $0.49-0.55$ & -0.28 & 3.9 \\
\hline & Avg staff & 7.1 & 7.1 & 0.87 & 0.73 & 0.85 & & & & \\
\hline \multirow[t]{2}{*}{ Portable equipment } & Obs & 6.7 & 7.3 & 0.07 & 0.93 & 0.96 & 0.25 & $0.23-0.26$ & -2.10 & 23.0 \\
\hline & Avg staff & 8.9 & 9.1 & 0.61 & 0.59 & 0.74 & & & & \\
\hline \multirow[t]{2}{*}{ Sedentary equipment } & Obs & 1.5 & 1.8 & 0.07 & 0.91 & 0.95 & 0.43 & $0.41-0.45$ & -0.73 & 31.4 \\
\hline & Avg staff & 2.3 & 2.3 & 0.88 & 0.78 & 0.87 & & & & \\
\hline \multirow[t]{2}{*}{ Inside space for gross motor activity $(1-5)$} & Obs & 3.3 & 3.3 & 0.83 & 0.98 & 0.99 & 0.03 & $0.02-0.06$ & 0.10 & 3.1 \\
\hline & Avg staff & 3.2 & 3.3 & 0.90 & 0.53 & 0.69 & & & & \\
\hline
\end{tabular}

$P$-values represent a statistical comparison of the equivalence of means for each day of reporting (means on days $1-4)$ for each reporter. $P$-values $>0.05$ indicate no statistical difference between means across days

ICC Intraclass correlation,

Corr Pearson correlation

Dev Difference (observation - staff report) 
Table 6 Nutrition and physical activity social environment - means, reliability, and validity

\begin{tabular}{|c|c|c|c|c|c|c|c|c|c|}
\hline \multirow[b]{2}{*}{ Staff behavior } & \multirow[b]{2}{*}{ Source } & \multirow[b]{2}{*}{$\begin{array}{l}\text { Means (range } \\
\text { day 1-4) }\end{array}$} & \multirow[b]{2}{*}{$\begin{array}{l}p \text {-value } \\
\text { means }\end{array}$} & \multicolumn{2}{|c|}{ Reliability } & \multicolumn{4}{|c|}{ Validity } \\
\hline & & & & $\mathrm{ICC} 1$ & ICC4 & $\begin{array}{l}\text { Corr } \\
\text { (avg) }\end{array}$ & Corr (days) & $\begin{array}{l}\text { Avg } \\
\text { dev }\end{array}$ & $\begin{array}{l}\text { Dev as } \\
\% \text { of mean }\end{array}$ \\
\hline \multicolumn{10}{|l|}{ During a meal/snack, the classroom teacher... } \\
\hline \multirow[t]{2}{*}{ Ate/drank an unhealthy food or beverage } & Obs & $1.18-1.70$ & 0.37 & 0.47 & 0.78 & & & & \\
\hline & Avg staff & $0.90-1.09$ & 0.75 & 0.47 & 0.78 & 0.26 & $0.02-0.45$ & 0.58 & 56.9 \\
\hline \multirow[t]{2}{*}{ Taught children about food eating } & Obs & $0.60-0.76$ & 0.66 & 0.39 & 0.72 & & & & \\
\hline & Avg staff & $0.46-0.86$ & 0.02 & 0.21 & 0.51 & 0.16 & $0.10-0.22$ & -0.03 & 4.1 \\
\hline \multirow[t]{2}{*}{ Sat with the children } & Obs & $1.02-1.42$ & 0.33 & 0.48 & 0.79 & & & & \\
\hline & Avg staff & $1.53-1.78$ & 0.47 & 0.51 & 0.81 & 0.28 & $0.21-0.36$ & -0.36 & 22.1 \\
\hline \multirow[t]{2}{*}{ Ate the same food as the children } & Obs & $1.06-1.44$ & 0.21 & 0.44 & 0.76 & & & & \\
\hline & Avg staff & $1.06-1.18$ & 0.90 & 0.60 & 0.86 & 0.46 & $0.38-0.53$ & 0.30 & 26.1 \\
\hline \multirow[t]{2}{*}{ Ate fruit or vegetables in front of children } & Obs & $0.60-0.90$ & 0.24 & 0.35 & 0.68 & & & & \\
\hline & Avg staff & $0.66-0.86$ & 0.47 & 0.39 & 0.72 & 0.39 & $0.30-0.45$ & 0.05 & 6.4 \\
\hline \multirow[t]{2}{*}{ Encouraged a child to eat more (3 items) } & Obs & $0.46-0.56$ & 0.94 & 0.23 & 0.55 & & & & \\
\hline & Avg staff & $0.61-1.21$ & 0.03 & 0.33 & 0.67 & 0.004 & $-0.11-0.04$ & -0.49 & 45.4 \\
\hline \multicolumn{10}{|l|}{ During play time, the... } \\
\hline \multirow{2}{*}{$\begin{array}{l}\text { Teacher joined children active play } \\
\text { ( } 3 \text { items) }\end{array}$} & Obs & $1.36-1.88$ & 0.42 & 0.32 & 0.48 & & & & \\
\hline & Avg staff & $0.52-0.71$ & 0.10 & 0.43 & 0.75 & 0.42 & $0.22-0.50$ & -0.05 & 11.4 \\
\hline \multirow[t]{2}{*}{ Teacher read a book promoting activity } & Obs & $0.04-0.10$ & 0.62 & -0.14 & -0.33 & & & & \\
\hline & Avg staff & $0.17-0.21$ & 0.89 & 0.26 & 0.58 & 0.11 & $-0.01-0.39$ & - & - \\
\hline \multirow[t]{2}{*}{ Children lost outside time (3 items) } & Obs & $0.05-0.09$ & 0.41 & 0.29 & 0.46 & & & & \\
\hline & Avg staff & $0.05-0.13$ & 0.01 & 0.16 & 0.44 & 0.25 & $0.07-0.35$ & -0.01 & 7.3 \\
\hline \multirow{2}{*}{$\begin{array}{l}\text { Children received extra outside time } \\
\text { ( } 2 \text { items) }\end{array}$} & Obs & $0-0$ & - & - & - & & & & \\
\hline & Avg staff & $0.02-0.05$ & 0.74 & 0.16 & 0.43 & - & - & - & - \\
\hline \multirow[t]{2}{*}{ Children lost TV time (2 items) } & Obs & $0.00-0.01$ & 0.51 & -0.10 & -0.22 & & & & \\
\hline & Avg staff & $0.01-0.02$ & 0.46 & 0.35 & 0.69 & 0.16 & $-0.02-0.26$ & -0.01 & 38.4 \\
\hline \multirow[t]{2}{*}{ Children received extra TV time (2 items) } & Obs & $0.00-0.01$ & 0.35 & - & - & & & & \\
\hline & Avg staff & $0.00-0.01$ & 0.72 & -0.04 & -0.17 & 0.47 & $0.00-0.70$ & - & - \\
\hline
\end{tabular}

$P$-values represent a statistical comparison of the equivalence of means for each day of reporting (means on days 1-4) for each reporter. $P$-values $>0.05$ indicate no statistical difference between means across days

ICC intraclass correlation,

Corr Pearson correlation,

Dev Difference (observation - staff report)

Table 7 Nutrition and physical activity policies - means, reliability and validity

\begin{tabular}{|c|c|c|c|c|c|c|c|c|}
\hline & \multicolumn{5}{|l|}{ Reliability } & \multicolumn{3}{|l|}{ Validity } \\
\hline & $\begin{array}{l}\text { Mean T1 } \\
\text { (SD) }\end{array}$ & $\begin{array}{l}\text { Mean T2 } \\
\text { (SD) }\end{array}$ & P_value & ICC1 & ICC2 & Mean Obs & $\begin{array}{l}\text { Corr } \\
\text { (T1 - Obs) }\end{array}$ & $\begin{array}{l}\text { P_value } \\
\text { (T1 - Obs) }\end{array}$ \\
\hline \multicolumn{9}{|c|}{ Total nutrition policies } \\
\hline Written & $7.49(6.29)$ & $8.58(7.70)$ & 0.61 & 0.76 & 0.86 & $6.72(5.40)$ & 0.27 & 0.67 \\
\hline General practice & $12.20(6.34)$ & $12.40(6.92)$ & 0.89 & 0.70 & 0.83 & & & \\
\hline \multicolumn{9}{|c|}{ Total physical activity policies } \\
\hline Written & $8.09(6.24)$ & $8.89(7.51)$ & 0.58 & 0.87 & 0.93 & $4.57(5.06)$ & 0.27 & 0.04 \\
\hline General practice & $10.96(6.01)$ & $11.33(7.74)$ & 0.80 & 0.79 & 0.88 & & & \\
\hline
\end{tabular}

$P$-values represent a statistical comparison of the equivalence of means for each day of reporting (means on days $1-4)$ for each reporter. $P$-values $>0.05$ indicate no statistical difference between means across days 
about a quarter of teachers (28\%) held a bachelor's degree or higher.

\section{Provisions: foods and beverages served}

Means, reliability and validity estimates for food and beverages served are presented in Table 3. Foods such as grains were served at each meal or snack occasion, while other foods such as fresh fruit, potatoes, and dessert were offered less frequently. Reliability estimates from the teacher report varied, with ICC estimates ranging from 0 to 0.96 , and were generally similar to estimates from the observation. Four-day intraclass correlations $\left(\mathrm{ICC}_{4}\right)$ indicate acceptable (fair or better) reliability for most foods after 4 days of reporting. Reliability estimates for potatoes, vegetables, dessert, and whole grains remained low for both observation and teacher report. Correlations between the observation and staff report, ranged from 0.25 to 0.85 , with a median of 0.52 . Milk, water, and potatoes had the highest correlations $(>0.6)$, while meat and grains had the lowest $(<0.33)$.

\section{Provisions: active and sedentary opportunities}

Results for PA and sedentary opportunities are presented in Table 4. On average, children were provided with about 60 min of outside time daily. Reporting of time outside was most consistent needing only 1 day of reporting to obtain acceptable reliability estimates. Most items had ICC estimates greater than 0.50 using 4 days of reporting. Correlations between the classroom teacher reports and the observation of PA and sedentary opportunities were fair to moderate. Stronger relationships were found for outside time, and TV time. Larger deviations between observations and reporting, as well as lower correlations, were found for teacher-led minutes of PA outside and seated minutes, with teachers over reporting teacher-led minutes of PA outside and underreporting seated minutes.

\section{Provisions: physical activity equipment and natural environment}

Table 5 presents results around physical activity equipment and natural environment features. One-day reliability estimates were high for fixed PA, portable PA, and sedentary equipment, as well as natural environment items. Staff and directors over reported natural, portable, and sedentary equipment by about $30 \%$ compared to the observation. The greatest difference was seen for natural elements, but the correlation between the two measures was high (0.70). For portable PA equipment, the difference between reported and observed was about $20 \%$, and the correlation between staff and observation was low (0.25), although this small difference (about 2 pieces of equipment) may not be practically meaningful.

\section{Practices: nutrition and physical activity social environment}

Results for the nutrition and physical activity social environment are presented in Table 6. Briefly, unhealthy practices such as teacher consumption of fast food, candy, or donuts were infrequently reported, while healthier practices like teachers eating the same food as the children or sitting with the children during meals were reported on average about once a day.

One- and 4-day reliability estimates ranged from 0.06 to 0.92 , with most above 0.3 . Validity estimate showed moderate to high agreement between the classroom teacher and observational reports. Higher correlations were seen for the healthier teacher practices, while teacher consumption of fast food and salty snacks had lower correlations between teacher and observation report.

\section{Policies: nutrition and physical activity}

Table 7 presents the means, reliability, and validity estimates for nutrition and physical activity policies. Directors reported whether the policy existed in their center as a formal, written policy or as general practice but not codified into formal policy. More common policies included types of food, beverages, amount of fruit and vegetables served, amount of active time and outdoor play time provided, appropriate clothes and shoes for outdoor play, staff behavior during outdoor play time, and amount/type of TV watched. Less common policies were generally the more in depth policies, such as cooking method for vegetables, promoting staff use of informal nutrition discussion during meals, amount and type of portable play equipment, and nutrition and PA education for parents. High percent agreement and Kappa statistics indicate adequate reliability for most policies, with Kappa statistics ranging from 0.23 to 0.82 , and most above 0.5 . Validity estimates were higher for policies around amount of fruit and vegetables served, family style dining, food brought by staff, informal nutrition discussion, and amount of time children can spend on computers. Validity estimates were on average higher for nutrition policies than PA policies. Table 7 shows that centers had policies covering about 20 of the 30 nutrition policy topic areas and about 19 of the 26 PA topic areas, with consistent reporting after 1 day of administration and moderate correlation with the observation report.

\section{Discussion}

The EPAO-SR instrument is an important advancement in the measurement of nutrition and physical activity environments in child care settings. To the best of our knowledge, no existing measure of the child care environment is as comprehensive or has undergone as rigorous development and evaluation as the EPAO-SR. The EPAO is based on a comprehensive review of the best practices for 
nutrition and physical activity environments at child care [19, 20-23]. The EPAO-SR incorporated a careful review of the existing EPAO instrument and included updates based on the current research literature, using cognitive interviews to ensure item clarity. Additionally, evaluation of this new instrument was carefully designed to provide evidence of test-retest reliability and validity of selfreport compared to 4 days of direct observation by trained staff. This ensures the quality of the instrument and strengthens the confidence in the findings for assessing the child care environment with the EPAO-SR. The EPAO-SR, in comparison to the other three existing instruments, is a more comprehensive self-assessment tool and with rigorous testing to establish multiple types of reliability and validity evidence.

Data from the current study demonstrated a range of reliability and validity evidence for the individual items contained in the EPAO-SR instrument. Results indicate that reporting from directors and classroom teachers is fairly consistent and similar to the observational data with only one or two administration needed to obtain minimally acceptable reporting of most items. Items that produced strongest reliability and validity estimates included beverages served, outside time, and physical activity equipment. These are factors within the child care environment that are more consistent across days and easier for child care staff to report; for example, physical activity fixed equipment is not likely to change from 1 day to the next and outside time may be a standard amount each day.

Although efforts were made to create the strongest instrument possible, reliability and validity evidence for some items appear low which seems troubling. However, reliability and validity evidence is not contained within a single number and instead is a function both of the item and the type of data collected. Low values $(\mathrm{ICC}<0.50)$ certainly help to identify problem areas that may need improvement, but also may identify true lack of variation between people or, in our case, centers. Whole grains served and amount of teacher-led PA had lower reliability and validity estimates. For these items, the reliability estimates were often similar for the child care staff report compared with the observation report. The low reliability or validity estimates may reflect the variable nature of those factors (i.e., day-to-day variability) and/or the inherent difficulties in measuring them, rather than inconsistent or misreporting by child care staff. Additionally, low ICC and correlation estimates can result from low between-subject variation, even with low within-subject variation. In this study, for example, grains were offered at nearly every meal at the majority of centers. This lack of variation likely contributed to the low validity estimate for total grains, since the means across the 4 days were similar between the classroom teachers and between the observation days. Because of these challenges, more days measured or more teachers reporting could improve the estimates for these items.

Differences in reporting also may have occurred due to the subjective nature of some factors, such as teacher behavior during mealtimes and active play. Other items, for example, teachers eating fast food, may have occurred during nap when observers were unable to note this, as observers typically left the center during nap time. Reporting bias by staff may have contributed to the differences noted between certain self-report items and observation data. It is not uncommon for individuals to over report to present a more favorable picture of their behavior. Interesting, we have also seen evidence of underreporting in areas where teachers feel they lack training or low selfefficacy (e.g., support for healthy eating). Although it may be impossible to eliminate bias reporting, survey instructions which stress "no-fault" reporting or "no right answer" may improve accuracy.

Some of the low validity estimates could be strengthened in future administrations by modifying the response format for particular items. For example, in the area of teacher-led bouts of physical activity, correlations between observation and self-report were low, and the mean differences were large. In the EPAO-SR, staff were asked to report the number of teacher-led physical activity bouts in an open response format. This resulted in some higher than expected values. As a modification we have limited the response format so that teachers can select $0-5+$ for this item, since more than $90 \%$ of the responses fell within this range. The categorical response seems to be easier for teachers to report and increases the reliability and validity estimates for these items. Thus, changes to instructions and limiting upper boundaries of behavior reporting could improve data quality by reducing burden caused by extreme overreporting.

Finally, some items, including portable play equipment, may have been stored in areas that were inaccessible to the observer, e.g., stored in another classroom but available for use by multiple classrooms. In this case, the classroom teacher may have reported the presence (availability) of portable play equipment whereas the observer would not have. Continued work may be necessary to address some of these problems and improve upon the instrument.

Although the EPAO-SR was based on an extensive review of authoritative recommendations and research literature, some aspects of the nutrition and physical activity child care environments may need modification based on emerging literature. A few additional areas were noted and will be incorporated into the next version of the EPAO-SR. These areas include more items within staff behavior (enthusiastic role modeling [38-41], verbal praise [41-44], authoritative feeding practices [43-46], and expanded details on the outdoor learning environment, including use of paved, curved pathways and available 
shade [47, 48], and playground density (child-to-space ratio) [49].

While the sample of child care staff and children in the centers was representative of the racial distribution in North Carolina, the sample was moderately homogenous with $50-60 \%$ of the sample (including directors, classroom teachers, and children) reporting as Non-Hispanic White. Also, the median star rating for the observed centers was high (4 out of 5). While nutrition and physical activity standards are not incorporated in the star rating system, the sample was from centers with higher child care quality standards. The EPAO-SR would benefit from additional testing in more diverse populations, including centers with lower quality ratings, from rural communities, and centers located in lower income neighborhoods. Additionally, there is no observational "gold standard" for some of the measured constructs, including directors' confidence to make healthy changes within the center and classroom teachers' valuation of nutrition and physical activity. These are important factors that can influence the child care environment and children's behaviors, but more work is needed to strength the construct validity evidence for these measures.

The purpose of the EPAO-SR is to evaluate the quality of the child care center's nutrition and physical activity environments. It uses data gathered from different sources to produce center-level scores. The Director Reportand the Staff General Questionnaire are executed with a single administration. The Staff Daily Questionnaire should be administered by a single teacher on two separate days or by two teachers during a single day. Information from the multiple administrations of the Staff Daily Questionnaire (either more days or more teachers) should be averaged. Finally, the data from the two general questionnaires and the average scores from the daily questionnaire are used to represent the center's nutrition and PA environments. Because this is a center level assessment, a multi-level model is not appropriate for summarization of these data. Because all children are served the same food and play on the same playground, the overall burden for reporting can be shared by staff and director, and aspects of the center can be evaluated by the most knowledgeable individuals. Because of clustering, different teachers can report food each day decreasing overall burden, while maintaining the quality of the measurement. If there is interest in using the EPAO-SR for classroom level practices and provisions, modifications would be necessary to the protocol to obtain classroom-level estimates instead of center-level.

We encourage use of this instrument by others in the field, either in its entirety or subsections based on individual needs (e.g., only assessing food and beverage provisions.) The use of standardized measures across different studies will strengthen our knowledge of the complex child care nutrition and physical activity environments, as comparisons between studies is easier when similar measures are used. As initially developed, the EPAO was used with a single day administration. Results from this study indicate that, when using the EPAO (observation), multiple days of observation (minimum of two) should be used to improve the validity and stability of the factors within the child care environment. As noted in Table 8, we recommend that the director and at least two teachers be used to obtain the full measure of the nutrition and PA environments within early care and education settings. The Director Report and Staff General Questionnaires can be administered only once, but the Staff Daily Questionnaire requires a minimum of two teachers reporting, or one teacher reporting on 2 days. Optimal practice would be having one teacher report for multiple days (3-4), or multiple teachers reporting for 2-3 days. If fewer days and reporters are employed, the risk will be the loss of information in areas where the occurrence of the provision or behavior is less frequent, difficult to assess, or very similar across ECEs. These decisions will depend upon the needs of the study and researchers.

Since the implementation of this project, we have continued to update and modify the original observationbased EPAO and the EPAO-SR to reflect current NAP SACC best practices and to expand the instrument's use to other child care settings (e.g., family child care homes). In addition, a scoring system for the EPAO-SR is being developed, similar to that of the initial EPAO observation which will be available along with a copy of the EPAO-SR instrument upon request. We do not necessarily suggest that the EPAO-SR (or even the EPAO in its observational form) is an instrument that should be used for licensing, center-specific funding, or compliance to state or federal regulations. To our knowledge, there are no instruments available which we would recommend for these purposes. Observation is often thought of as a gold standard and may be better for "high-stakes" use in some cases, but we have found that the reliability evidence for observation is similar to teacher and director report in most cases. The EPAO and EPAO-SR are research quality instruments, which can be used to evaluate interventions, test theory,

Table 8 Recommendations for future use

\begin{tabular}{lll}
\hline Reporter & Content & $\begin{array}{l}\text { Days of } \\
\text { reporting }\end{array}$ \\
\cline { 3 - 3 } & & Minimal \\
\hline $\begin{array}{l}\text { Classroom } \\
\text { teacher }\end{array}$ & Provisions (Nutrition and PA) \\
& a) Foods & 2 days $\geq 4$ days \\
& b) Beverages & 1 day $\quad 2$ days \\
& c) PA and sedentary time & 2 days $\geq 4$ days \\
& d) Physical environment & 1 day $\geq 2$ days \\
Center & Practices (Nutrition and PA) & 1 day 2 days \\
director & Policies, training, and education & 1 day 1 day \\
\hline
\end{tabular}

${ }^{a}$ Multiple days of reporting could be distributed between 2 or more teachers 
and aggregate center level data to inform policy makers about needed changes or to examine the impact of policy change. We hope that these efforts dedicated to improving the measurement of child care settings will facilitate continued work in this important area.

\section{Conclusions}

Overall, this instrument offers many benefits to the field and can be used in a variety of formats by both researchers and practitioners. A recent meeting of early care and education experts underscored the need for these methods to be "translated and simplified to facilitate use by others," which is a central goal of the development of this instrument [16]. The EPAO-SR fills this need, as it is both a lowcost and a low-burden tool. The cost of a self-report is much lower than the cost of the traditional gold standard of observation, and dividing the survey amongst directors and classroom teachers reduces the burden of reporting. Additionally, the tool can be used to characterize the child care environment, to understand the relationship between aspects of the child care environment and child weightrelated behaviors, and to evaluate interventions targeting the child care environment.

\section{Competing interests}

The authors declare that they have no competing interests.

\section{Authors' contributions}

DW served as the Principal Investigator for the project, with DH serving as co-investigator. $\mathrm{CM}, \mathrm{DH}$, and DW participated in reviewing the literature and retrieval and creation of items. CM oversaw cognitive interviews and summarization of interpretability issues, incorporating feedback from $\mathrm{DH}$ and DW to improve item clarity. SM and CM oversaw collection of reliability and validity data. $\mathrm{DH}$ conducted analyses. $\mathrm{DH}, \mathrm{SM}, \mathrm{CM}$, and DW participated in data review and interpretation of analyses. SM and DW led manuscript development, with contributions, edits, and review by DH and CM. All authors read and approved the final manuscript.

\section{Acknowledgements}

The authors would like to thank the directors and teachers who participated in this study and the data collectors, Amanda Tillotson and Pairin Seepolmaung. The current research was funded by the Healthy Eating Research and Active Living Research programs of the Robert Wood Johnson Foundation (RWJF ID: 63050). The project was conducted out of the Center for Health Promotion and Disease Prevention, a Prevention Research Center funded through a cooperative agreement with the Centers for Disease Control and Prevention (U48-DP001944). We are also grateful for the additional support for this project, from the NIDDK grant P30DK056350 to the UNC Nutrition Obesity Research Center and the NIH/ NINR grant T32NR007091 Interventions for Preventing \& Managing Chronic Illness (PI: S. Santacroce). The content is solely the responsibility of the authors and does not necessarily represent the official views of the Centers for Disease Control and Prevention, NIDDK or the $\mathrm{NIH}$.

\section{Author details}

'Department of Nutrition, Gillings School of Global Public Health, University of North Carolina at Chapel Hill, 2207 McGavran-Greenberg Hall, CB 7461, Chapel Hill, NC 27599-7461, USA. ${ }^{2}$ Department of Nutrition, Gillings School of Global Public Health, and Center for Health Promotion and Disease Prevention, University of North Carolina at Chapel Hill, 1700 Martin L. King Jr. Blvd., CB 7426, Chapel Hill, NC 27599-7426, USA. ${ }^{3}$ Center for Health Promotion and Disease Prevention, University of North Carolina at Chapel Hill, 1700 Martin L. King Jr. Blvd., CB 7426, Chapel Hill, NC 27599-7426, USA.
Received: 13 March 2015 Accepted: 17 September 2015

Published online: 26 September 2015

\section{References}

1. Ogden CL, Carroll MD, Kit BK, Flegal KM. Prevalence of obesity and trends in body mass index among US children and adolescents, 1999-2010. JAMA. 2012;307(5):483-90.

2. Singh AS, Mulder C, Twisk JW, van Mechelen W, Chinapaw MJ. Tracking of childhood overweight into adulthood: a systematic review of the literature. Obes Rev. 2008;9(5):474-88.

3. Dietz WH. Health consequences of obesity in youth: childhood predictors of adult disease. Pediatrics. 1998;101(3):518-25.

4. Freedman DS, Serdula MK, Srinivasan SR, Berenson GS. Relation of circumferences and skinfold thicknesses to lipid and insulin concentrations in children and adolescents: the Bogalusa Heart Study. Am J Clin Nutr. 1999;69(2):308-17.

5. Pinhas-Hamiel O, Dolan LM, Daniels SR, Standiford D, Khoury PR, Zeitler P. Increased incidence of non-insulin-dependent diabetes mellitus among adolescents. J Pediatr. 1996;128(5 Pt 1):608-15.

6. Schwimmer JB, Deutsch R, Kahen T, Lavine JE, Stanley C, Behling C. Prevalence of fatty liver in children and adolescents. Pediatrics. 2006;118(4):1388-93.

7. Dietz W. Overweight in childhood and adolescence. N Engl J Med. 2004;350(9):855-7.

8. Monteiro PO, Victora CG. Rapid growth in infancy and childhood and obesity in later life-a systematic review. Obes Rev. 2005;6(2):143-54.

9. Nader PR, O'Brien M, Houts R, Bradley R, Belsky J, Crosnoe R, et al. Identifying risk for obesity in early childhood. Pediatrics. 2006;118(3):E594-601.

10. Story M, Kaphingst KM, Robinson-O'Brien R, Glanz K. Creating healthy food and eating environments: policy and environmental approaches. Annu Rev Public Health. 2008;29:253-72.

11. Larson N, Ward DS, Neelon SB, Story M. What role can child-care settings play in obesity prevention? A review of the evidence and call for research efforts. J Am Diet Assoc. 2011;111(9):1343-62.

12. US Department of Education and National Center for Education Statistics. Early childhood program participation survey of the national household education surveys program. 2006.

13. Stang J, Bayerl CT. Position of the American Dietetic Association: child and adolescent food and nutrition programs. J Am Diet Assoc. 2003;103(7):887-93.

14. American Academy of Pediatrics. Caring for our children: National health and safty performance standards; guidelines for early care and education programs. Elk Grove Village: American Academy of Pediatrics; Washington: American Public Health Association; 2011.

15. Ball SC, Benjamin SE, Ward DS. Dietary intakes in North Carolina child-care centers: are children meeting current recommendations? J Am Diet Assoc. 2008;108(4):718-21.

16. Padget A, Briley ME. Dietary intakes at child-care centers in central Texas fail to meet Food Guide Pyramid recommendations. J Am Diet Assoc. 2005;105(5):790-3.

17. Reilly JJ. Low levels of objectively measured physical activity in preschoolers in child care. Med Sci Sports Exerc. 2010;42(3):502-7.

18. Ward DS, Vaughn A, Story M. Expert and stakeholder consensus on priorities for obesity prevention research in early care and education settings. Child Obes. 2013;9(2):116-24.

19. Ward D, Hales D, Haverly K, Marks J, Benjamin S, Ball S, et al. An instrument to assess the obesogenic environment of child care centers. Am J Health Behav. 2008;32(4):380-6.

20. Ward DS, Benjamin SE, Ammerman AS, Ball SC, Neelon BH, Bangdiwala SI. Nutrition and physical activity in child care: results from an environmental intervention. Am J Prev Med. 2008;35(4):352-6.

21. Ammerman AS, Ward DS, Benjamin SE, Ball SC, Sommers JK, Molloy M, et al. An intervention to promote healthy weight: Nutrition and Physical Activity Self-Assessment for Child Care (NAP SACC) theory and design. Prev Chronic Dis. 2007;4(3):A67.

22. Benjamin Neelon SE, Vaughn A, Ball SC, McWilliams C, Ward DS. Nutrition practices and mealtime environments of North Carolina child care centers. Child Obes. 2012;8(3):216-23.

23. McWilliams C, Ball SC, Benjamin SE, Hales D, Vaughn A, Ward DS. Bestpractice guidelines for physical activity at child care. Pediatrics. 2009;124(6):1650-9. 
24. Benjamin Neelon SE, Taveras EM, Ostbye T, Gillman MW. Preventing obesity in infants and toddlers in child care: results from a pilot randomized controlled trial. Matern Child Health J. 2014;18(5):1246-57.

25. Gubbels JS, Van Kann DH, Jansen MW. Play equipment, physical activity opportunities, and children's activity levels at childcare. J Environ Public Health. 2012;2012:326520.

26. Gubbels JS, Kremers SP, van Kann DH, Stafleu A, Candel MJ, Dagnelie PC, et al. Interaction between physical environment, social environment, and child characteristics in determining physical activity at child care. Health Psychol. 2011:30(1):84-90.

27. Bower JK, Hales DP, Tate DF, Rubin DA, Benjamin SE, Ward DS. The childcare environment and children's physical activity. Am J Prev Med. 2008;34(1):23-9.

28. Lyn R, Maalouf J, Evers S, Davis J, Griffin M. Nutrition and physical activity in child care centers: the impact of a wellness policy initiative on environment and policy assessment and observation outcomes, 2011. Prev Chronic Dis. 2013;10:E83.

29. Maalouf J, Evers SC, Griffin M, Lyn R. Assessment of mealtime environments and nutrition practices in child care centers in Georgia. Child Obes. 2013;9(5):437-45

30. Vanderloo LM, Tucker $\mathrm{P}$, Johnson AM, van Zandvoort MM, Burke SM, Irwin JD. The influence of centre-based childcare on preschoolers' physical activity levels: a cross-sectional study. Int J Environ Res Public Health. 2014;11(2):1794-802

31. Tucker $P$, Vanderloo LM, Newnham-Kanas C, Burke SM, Irwin JD, Johnson AM, et al. Learning Environments' Activity Potential for Preschoolers (LEAPP): study rationale and design. J Public Health Res. 2013;2(2):e19.

32. Gubbels JS, Kremers SP, Stafleu A, Dagnelie PC, de Vries NK, Thijs C. Childcare environment and dietary intake of 2- and 3-year-old children. J Hum Nutr Diet. 2010;23(1):97-101.

33. Falbe J, Kenney EL, Henderson KE, Schwartz MB. The Wellness Child Care Assessment Tool: a measure to assess the quality of written nutrition and physical activity policies. J Am Diet Assoc. 2011;111(12):1852-60.

34. Copeland KA, Sherman SN, Khoury JC, Foster KE, Saelens BE, Kalkwarf HJ. Wide variability in physical activity environments and weather-related outdoor play policies in child care centers within a single county of Ohio. Arch Pediatr Adolesc Med. 2011;165(5):435-42.

35. Henderson KE, Grode GM, Middleton AE, Kenney EL, Falbe J, Schwartz MB. Validity of a measure to assess the child-care nutrition and physical activity environment. J Am Diet Assoc. 2011;111(9):1306-13.

36. Willis GB. Cognitive interviewing: a tool for improving questionnaire design. Thousand Oaks: Sage Publications; 2005.

37. Shrout PE. Measurement reliability and agreement in psychiatry. Stat Methods Med Res. 1998;7(3):301-17.

38. Frazier BN et al. I'll have what she's having: the impact of model characteristics on children's food choices. Dev Sci. 2012;15(1):87-98.

39. Gibson EL, Kreichauf S, Wildgruber A, Vögele C, Summerbell CD, Nixon C, et al. A narrative review of psychological and educational strategies applied to young children's eating behaviours aimed at reducing obesity risk. Obes Rev. 2012;13 Suppl 1:85-95.

40. Hendy HM, Raudenbush B. Effectiveness of teacher modeling to encourage food acceptance in preschool children. Appetite. 2000;34(1):61-76.

41. Summerbell CD, Moore HJ, Vögele C, Kreichauf S, Wildgruber A, Manios $Y$, et al. Evidence-based recommendations for the development of obesity prevention programs targeted at preschool children. Obes Rev. 2012;13 Suppl 1:129-32.

42. Horne PJ, Hardman CA, Lowe CF, Tapper K, Le Noury J, Madden P, et al. Increasing parental provision and children's consumption of lunchbox fruit and vegetables in Ireland: the Food Dudes intervention.

Eur J Clin Nutr. 2009;63(5):613-8.

43. Murashima M, Hoerr SL, Hughes SO, Kaplowitz S. Confirmatory factor analysis of a questionnaire measuring control in parental feeding practices in mothers of Head Start children. Appetite. 2011;56(3):594-601.

44. Murashima M, Hoerr SL, Hughes SO, Kaplowitz SA. Feeding behaviors of low-income mothers: directive control relates to a lower BMI in children, and a nondirective control relates to a healthier diet in preschoolers. Am Clin Nutr. 2012;95(5):1031-7.

45. Berge JM. A review of familial correlates of child and adolescent obesity: what has the 21st century taught us so far? Int J Adolesc Med Health. 2009;21(4):457-83.
46. Hughes SO, Patrick H, Power TG, Fisher JO, Anderson CB, Nicklas TA. The impact of child care providers' feeding on children's food consumption. J Dev Behav Pediatr. 2007;28(2):100-7.

47. Cosco NG, Moore RC, Islam MZ. Behavior mapping: a method for linking preschool physical activity and outdoor design. Med Sci Sports Exerc. 2010;42(3):513-9.

48. Cosco NG, Moore RC, Smith WR. Childcare outdoor renovation as a built environment health promotion strategy: evaluating the preventing obesity by design intervention. Am J Health Promot. 2014;28(3 Suppl):S27-32.

49. Cardon G, Van Cauwenberghe E, Labarque V, Haerens L, De Bourdeaudhuij I. The contribution of preschool playground factors in explaining children's physical activity during recess. Int J Behav Nutr Phys Act. 2008:5:11.

\section{Submit your next manuscript to BioMed Central and take full advantage of:}

- Convenient online submission

- Thorough peer review

- No space constraints or color figure charges

- Immediate publication on acceptance

- Inclusion in PubMed, CAS, Scopus and Google Scholar

- Research which is freely available for redistribution 